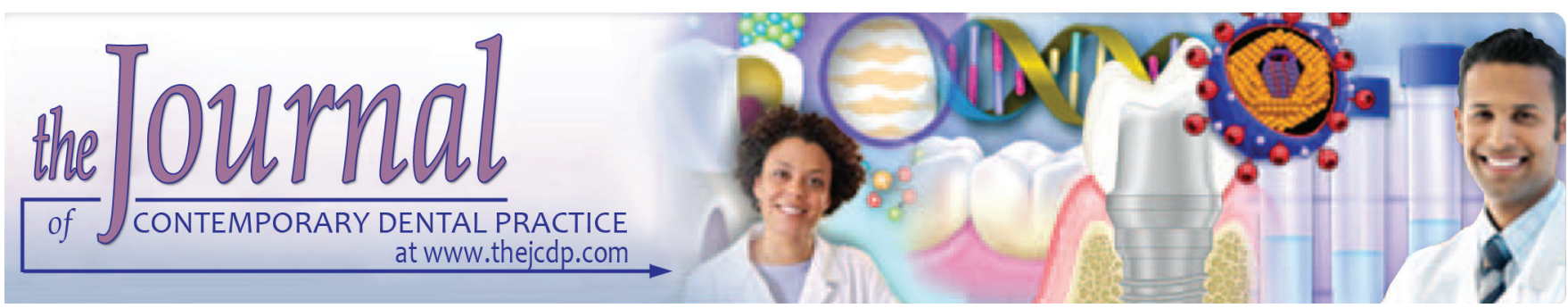

\title{
Evaluation of Antimicrobial Efficacy of Root Canal Sealers against Enterococcus faecalis: A Comparative Study
}

\author{
${ }^{1}$ Suraj Arora, ${ }^{2}$ Shugufta Mir, ${ }^{3}$ Ashesh Gautam, ${ }^{4}$ Renu Batra, ${ }^{5}$ Sarmeshta Soni, ${ }^{6}$ Kamini Lata
}

\begin{abstract}
Aim: The study aimed to evaluate the efficacy of root canal sealer's antimicrobial activity against Enterococcus faecalis.

Materials and methods: Root canal sealers with the brands Endoflas FS, AH Plus, and Tubli-Seal EWT were selected. Enterococcus faecalis organisms' zone of inhibition was measured. Tukey post hoc tests and one-way analysis of variance (ANOVA) were used to compare the data among study groups and within its group. The data were said to be statistically significant with an established $\mathrm{p}$-value less than 0.05 .
\end{abstract}

Results: The maximum zone of inhibition $(23.20 \pm 1.202 \mathrm{~mm})$ was shown by Endoflas FS sealer; AH Plus and Tubli-Seal showed the inhibition zone of $(18.42 \pm 1.023)$ and $(16.88 \pm$ $0.962)$ respectively, following Endoflas FS. The ANOVA test showed $p<0.0001$, which is highly statistically significant. $\mathrm{AH}$ Plus and Endoflas FS showed a statistically significant difference of 0.04 and 0.001 in between groups, and there was no statistically significant difference in the Tubli-Seal group from Tukey post hoc test.

Conclusion: The study showed that Endoflas FS sealer has a significant antimicrobial effect against $E$. faecalis.

Clinical significance: In clinical situation, despite perfect chemomechanical root canal preparation, persistence of microorganisms may reinfect the root canal. Therefore, endodontic root canal sealers play a major role in the eradication of bacteria. The polymicrobial nature of endodontic infection plays a main

\footnotetext{
1,2Department of Restorative Dentistry, College of Dentistry King Khalid University, Abha, Kingdom of Saudi Arabia

${ }^{3,5,6}$ Department of Pedodontics and Preventive Dentistry Awadh Dental College and Hospital, Jamshedpur, Jharkhand India

${ }^{4}$ Department of Conservative Dentistry and Endodontics Manubhai Patel Dental College \& Charitable Dental Hospital \& ORI, Vadodara, Gujarat, India

Corresponding Author: Suraj Arora, Department of Restorative Dentistry, College of Dentistry, King Khalid University, Abha Kingdom of Saudi Arabia, e-mail: surajarorasgrd@yahoo.co.in
}

role during the usage of endodontic sealer with antimicrobial agents, which in turn reduces the failure of endodontic treatment.

Keywords: AH Plus, Antimicrobial agent, Endodontic infections, Endoflas FS, Enterococcus faecalis, Tubli-Seal.

How to cite this article: Arora S, Mir S, Gautam A, Batra R, Soni S, Lata K. Evaluation of Antimicrobial Efficacy of Root Canal Sealers against Enterococcus faecalis: A Comparative Study. J Contemp Dent Pract 2018;19(6):680-683.

Source of support: Nil

Conflict of interest: None

\section{INTRODUCTION}

The primary causative agents of apical periodontitis and necrotic pulpitis are microorganisms and its by-products. Endodontic therapy is the only treatment to preserve the teeth; if not, the teeth need to be extracted. Elimination of the microorganisms and prevention of the infection subsequently are the main aims of endodontic therapy. ${ }^{1}$

The factors which affect the success of the endodontic therapy begin from access opening, biomechanical preparation, and three-dimensional obturation of root canal. One of the main factors for root canal treatment failure is the residual infection; therefore, complete eradication of the infection is necessary for the success of the endodontic treatment. $^{2}$

The microorganism most commonly found in endodontic infection is E. faecalis. This has been proved with its factor of survival and virulence, including the ability to invade dentinal tubules, resistance to deprived nutrition, and to compete with different microorganisms. ${ }^{3}$

The core aim of endodontic treatment is to obturate the root canal completely to obtain a sterile root canal, preventing bacteria to recontaminate and recolonize inside the root canal space. A bacteriostatic root canal sealer is required for the ideal treatment according to Grossman. ${ }^{4}$ Poor quality root canal filling leads to periodontal tissue breakdown. A tooth which is endodontically treated and 
has periapical pathology has an impaired or retarded subsequent healing capacity. To improve the antibacterial effect of sealer root canal therapy, antibacterial agents are added. Many sealers for root canal composed of calcium hydroxide, zinc oxide, and epoxy resin are available, yet only a few are effective to eradicate pathogens, particularly strict anaerobes. ${ }^{5}$

The infection is prevented by the root canal sealer by entombing survival bacteria, to block further microbial effects on the root canal and to avoid fluids of periapical tissue to reach bacterial cells inside the root canal and sustaining bacterial survival. ${ }^{6}$ Therefore, this study evaluates the antimicrobial effect of root canal sealers against E. faecalis.

\section{MATERIALS AND METHODS}

The study was conducted using three different root canal sealers. This in vitro study was conducted in Jan Nayak Choudhary Devi Lal Dental College, Haryana, India.

\section{Root Canal Sealers and Their Composition}

\section{AH Plus (Dentsply Detrey, USA)}

This sealer of epoxy resin base is a pack of two pastes, pastes A and B. Paste A contained bisphenol F epoxy resin, bisphenol A epoxy resin, zirconium oxide, calcium tungstate, silica, and pigments of iron oxide. Paste B contained tricyclodecane diamine, dibenzyldiamine, aminoadamantane, zirconium oxide, calcium tungstate, silica, and oil of silicone.

\section{Endoflas FS (Sanlor, Colombia)}

The sealer is composed of iodoform incorporated in zinc oxide eugenol powder. The powder is composed of zinc oxide, barium sulfate, calcium hydroxide, and liquid composed of eugenol and zinc acetate accelerator.

\section{Tubli-Seal EWT (Sybron Endo Corporation, Orange, $C A$ )}

The sealer consists of base paste and catalyst paste. The base paste is a zinc oxide eugenol base composed of zinc oxide, thymol iodide, bismuth trioxide, oils, and waxes. The catalyst paste is composed of eugenol, polymerized resins, and annidalin.

\section{Preparation of the Medium for E. faecalis}

The Mueller-Hinton ( $\mathrm{MH}$ ) agar medium was prepared and poured using $20 \mathrm{~mL}$ of sterilized pipettes into Petri dishes which are sterilized and on a flat horizontal surface up to a depth of $4 \mathrm{~mm}(20 \mathrm{~mL})$. The Petri dishes were stored in a refrigerator under $4^{\circ} \mathrm{C}$ which is used within 1 week from preparation.
The colonies of E. faecalis with similar morphology were touched using a sterile wore loop. The organisms were transferred into a sterile test tube prefilled with sterile brain heart infusion broth of $1.5 \mathrm{~mL}$. The test tube was incubated at $37^{\circ} \mathrm{C}$ for 24 hours, to cultivate a moderately turbid bacterial suspension. The bacterial suspension density is standardized, comparing its density with $0.5 \mathrm{McF}$ arland units of the barium sulfate standard. The standard is equivalent to $10^{8}$ colony-forming units per milliliter.

The $\mathrm{MH}$ agar culture medium was used for inoculation. A sterile cotton swab dipped in the bacterial suspension was taken, removing the excess by rolling the swab to the test tube side. The entire surface of the culture medium plate was streaked with the cotton swab in three directions evenly.

To investigate the root canal sealers' antimicrobial activity, the agar diffusion method is used. In suitable culture media, the subculture of the microorganism was made to check their purity. Three wells of equal size were made by extracting the agar at the same distance and filled the root canal sealers. The mixing of the sealer is done as per the manufacturer's instruction. Microorganisms are cultivated for 24 hours at $37^{\circ} \mathrm{C}$ in $\mathrm{MH}$ broth. The $\mathrm{MH}$ broth was incorporated as a second layer. This $\mathrm{MH}$ broth was added over after the placement of the sealer.

For prediffusion, the plates were kept for 2 hours at room temperature. The incubation was done at $37^{\circ} \mathrm{C}$ for 24 hours under suitable gaseous conditions. The diameter of the zone of inhibition was measured in millimeters around the plate after incubation.

Statistical Package for the Social Sciences software version 17.0 was used for data analysis. To compare the data between the groups and within the groups, Tukey post hoc tests and one-way ANOVA were used; $\mathrm{p}<0.05$ was considered statistically significant.

\section{RESULTS}

Table 1 shows the mean zone of inhibition E. faecalis within three sealers of root canal. The maximum zone of inhibition is shown by Endoflas FS $(23.20 \pm 1.202 \mathrm{~mm})$ which is followed by AH Plus (18.42 \pm 1.023$)$ and TubliSeal (16.88 \pm 0.962).

Table 2 shows the statistical comparison of the antimicrobial activity within the group and between the sealers

Table 1: Mean zone of inhibition of E. faecalis with three root canal sealers

\begin{tabular}{lll}
\hline & \multicolumn{2}{c}{ E. faecalis } \\
\cline { 2 - 3 } Root canal sealers & Mean & Standard deviation \\
\hline AH Plus & 18.42 & 1.023 \\
Endoflas FS & 23.20 & 1.202 \\
Tubli-Seal & 16.88 & 0.962 \\
\hline
\end{tabular}


Table 2: Comparison of zone of inhibition among root canal sealers using ANOVA

\begin{tabular}{llllll}
\hline & $\begin{array}{l}\text { Sum of } \\
\text { squares }\end{array}$ & Df & $\begin{array}{l}\text { Mean } \\
\text { square }\end{array}$ & f-value & Sig. \\
\hline Between groups & 9133.221 & 5 & 1108.542 & 2264.520 & 0.0001 \\
Within groups & 16.100 & 46 & 0.522 & & \\
\hline Total & 9149.321 & 51 & & & \\
\hline
\end{tabular}

Table 3: Within-group comparison between different root canal sealers with $E$. faecalis

\begin{tabular}{llll}
\hline Root canal sealers & E. faecalis & Standard error & $p$-value \\
\hline AH Plus & $18.42 \pm 1.023$ & 0.1432 & 0.04 \\
Endoflas FS & $23.20 \pm 1.202$ & 0.1140 & 0.001 \\
Tubli-Seal & $16.88 \pm 0.962$ & 0.021 & 0.06 \\
\hline
\end{tabular}

tested using ANOVA, which is found to be $p<0.0001$, which is statistically highly significant.

Table 3 depicts the comparison between the sealers' activity against E. faecalis. Endoflas FS and AH Plus sealers projected a difference of 0.001 and 0.04 between the groups respectively, which is statistically significant. There was no significant difference in the Tubli-Seal sealer.

\section{DISCUSSION}

The outcome of the endodontic treatment depends on the complete eradication of the bacteria from the root canal, and to provide an efficient seal to avoid further reinfection. Enterococcus faecalis was selected in this study mainly because of its possible activity in the endodontic infection. ${ }^{7}$

This study results showed that Endoflas FS projected the antimicrobial activity which was higher when compared with AH Plus and Tubli-Seal, which had the least activity against $E$. faecalis as that of Saha et $\mathrm{al}^{8}$ and Gomes et $\mathrm{al}^{9}$ study.

The antimicrobial activity was highest in Endoflas FS mainly because of its content iodoform. Iodoform is added in modified zinc-oxide eugenol sealers, such as medicated canal sealer, and Endoflas FS to improve its antimicrobial property. Iodoform has a mild antiseptic property, as it releases iodine slowly when it is exposed to other body fluids. The iodine content makes it fungicidal and bactericidal. The mechanism of action of the iodoform is to release its oxidizing agent iodine, which can oxidize irreversibly and inactivate vital metabolic compounds, such as a protein by its antimicrobial action. This result is similar to that of the study conducted by Kayaoglu et $\mathrm{al}^{10}$ and Aravind et al. ${ }^{11}$

Anaerobic bacteria can sustain in the environment without oxygen and with minimal nutrients. As the root canal is a closed place, with less or no oxygen, it makes it an ideal environment to harbor anaerobic bacteria. The environmental condition in the root canal, such as bacterial relationships, oxygen tension, and nutrients lays a platform for the anaerobic bacteria to ferment peptides and amino acids. The bacteria that mainly extract energy from carbohydrate fermentation are restricted by lack of nutrients. ${ }^{12}$

The antimicrobial activity was higher in AH Plus which is freshly mixed when compared with Tubli-Seal, especially against $E$. faecalis. The present study results supported these findings; the antimicrobial activity against E. faecalis in AH Plus was better than Tubli-Seal, which is eugenol based. AH Plus showed to release a few amine ingredients, epoxy, and formaldehyde during its polymerization process. Other contributing factors which back AH Plus sealers' performance are speculated. ${ }^{13}$

The mechanism of antimicrobial activity is by the speed of its dissociation into the hydroxyl and calcium ions. The hydroxyl ion dissociation creates an environment with high $\mathrm{pH}$, inhibiting enzymatic activities that are necessary for cellular division, growth, and metabolism of the microorganism. From the present study, AH Plus showed antimicrobial activity toward E. faecalis. But as per Mickel et al's ${ }^{14}$ study results, $\mathrm{AH}$ Plus did not show any zone of inhibition toward $E$. faecalis. Elimination of the formaldehyde release from $\mathrm{AH}$ Plus sealant led it to become ineffective against microorganisms.

Agar dilution and agar diffusion methods were used traditionally to detect the susceptibility for microorganisms. In this study, the agar disk diffusion method, called Kirby-Bauer method, was used instead of traditional agar dilution method. The agar dilution method's disadvantage is that this method is tested to alter few root canal sealers' properties. Additionally, few sealers cannot be dissolved homogenously, and it is a slow and difficult technique. Therefore, the agar disk diffusion method is used, as it does not change the sealer's chemical property. ${ }^{15}$

Siren et $\mathrm{al}^{16}$ presented the probable reasons for the failure of endodontically treated teeth due to the presence of E. faecalis, such as

- The presence of enteric bacteria in small amount inside the infected canal initially and increase in their proportion relatively during treatment, as other microorganisms are vulnerable to the therapy

- The entry of enteric bacteria during the root canal treatment is because of

- leaking of temporary filling

- working area's inadequate isolation

- opened root canal left for abscess drainage

Therefore, the ideal root canal treatment's objective is not only to eliminate infection but also to prevent reinfection of the treated root canal.

The rationale to perform this in vitro test is to provide valued information to the clinicians related to the various 
root canal sealers. Accordingly, an in vitro test is essential to determine the true effectiveness of the antimicrobial activity. Findings from this study showed that the endodontic sealers vary by the zone of inhibition of their antimicrobial activity.

\section{CONCLUSION}

The conclusion defines that Endoflas FS root canal sealer showed a significant increase in the antimicrobial activity against E. faecalis. The endodontic sealer's antibacterial property is an essential factor in preventing the growth of the bacteria in the root canal.

\section{REFERENCES}

1. Arora RS, Rawat P, Bhayya DP. A comparative evaluation of antimicrobial efficacy of three endodontic sealers: Endoflas FS, AH Plus and sealapex against Enterococcus faecalis-an in vitro study. IOSR J Dent Med Sci 2014 Mar;13(3):90-93.

2. Radwan IN, Randa B, Hend AN, Camilia G. Evaluation of antimicrobial efficacy of four medicinal plants extracts used as root canal irrigant on Enterococcus faecalis: an in-vitro study. Int Dent Med J Adv Res 2015;1:1-8.

3. Zehnder M. Root canal irrigants. J Endod 2006 May;32(5): 389-398.

4. Cavalcanti AL, Limeira FI, Sales EA, Oliveira AA, Lima DM, Castro RD. In vitro antimicrobial activity of root canal sealers and calcium hydroxide paste. Contemp Clin Dent 2010 Jul;1(3):164-167.

5. Mali S, Dhamali D, Desai A, Singh A, Thakur A, Shetty RR. Antimicrobial efficacy of different root canal sealers. Int J Oral Care Res 2016 Apr-Jun;4(2):131-133.

6. Hoelscher AA, Bahcall JK, Maki JS. In vitro evaluation of the antimicrobial effects of a root canal sealer-antibiotic combination against Enterococcus faecalis. J Endod 2006 Feb;32(2):145-147.

7. Sato I, Ando-Kurihara N, Kota K, Iwaku M, Hoshino E. Sterilization of infected root-canal dentine by topical application of a mixture of ciprofloxacin, metronidazole and minocycline in situ. Int Endod J 1996 Mar;29(2):118-124.

8. Saha S, Saha S, Samadi F, Jaiswal JN, Ghoshal U. An in vitro evaluation of antimicrobial activity of different endodontic sealers. JIDMR 2010 Dec;3(3):108-115.

9. Gomes BP, Pedroso JA, Jacinto RC, Vianna ME, Ferraz CC, Zaia AA, de Souza-Filho FZ. In vitro evaluation of the antimicrobial activity of five root canal sealers. Braz Dent J 2004;15(1):30-35.

10. Kayaoglu G, Erten H, Alacam T, Orstavik D. Short term antibacterial activity of root canal sealers towards Enterococcus faecalis. Int Endod J 2005 Jul;38(7):483-488.

11. Aravind, Gopikrishna V, Kandaswamy D, Jeyaval RK. Comparative evaluation of the antimicrobial efficacy of five endodontic root canal sealers against Enterococcus faecalis and Candida albicans. J Cons Dent 2006;9(1):2-11.

12. Peciuliene V, Balciuniene I, Eriksen HM, Haapasalo M. Isolation of Enterococcus faecalis in previously root-filled canals in a Lithuanian population. J Endod 2000 Oct;26(10):593-595.

13. Leonardo MR, Bezerra da Silva LA, Filho MT, Santana da Silva R. Release of formaldehyde by 4 endodontic sealers. Oral Surg Oral Med Oral Pathol Oral Radiol Endod 1999 Aug;88(2):221-225.

14. Mickel AK, Nguyen TH, Chogle SM. Antimicrobial activity of endodontic sealers on Enterococcus faecalis. J Endod 2003 Apr;29(4):257-258.

15. PumarolaJ, Berastegui E, BrauE,CanaldaC,JimenezdeAnta MT. Antimicrobial activity of seven root canal sealers. Oral Surg Oral Med Oral Pathol 1992 Aug;74(2):216-220.

16. Siren EK, Haapasalo MP, Ranta K, Salmi P, Kerosuo EN. Micro-biological findings and clinical treatment procedures in endodontic cases selected for microbiological investigation. Int Endod J 1997 Mar;30(2):91-95. 